\title{
II. Eine neue Art magnetischer und electrischer
}

Messinstrumente; von G. Quincke.

a. Magnetometer, Tangentenbussole und Multiplicator.

(Vorgezeigt der Gesellschaft deutscher Naturforscher und Aerzte in Halle am 22. September 1891.)

Als Magnetometer, Tangentenbussole oder Multiplicator benutze ich ein Messinstrument, welches im Wesentlichen aus Glas, Hartgummi und Holz besteht, keine Schrauben ent-

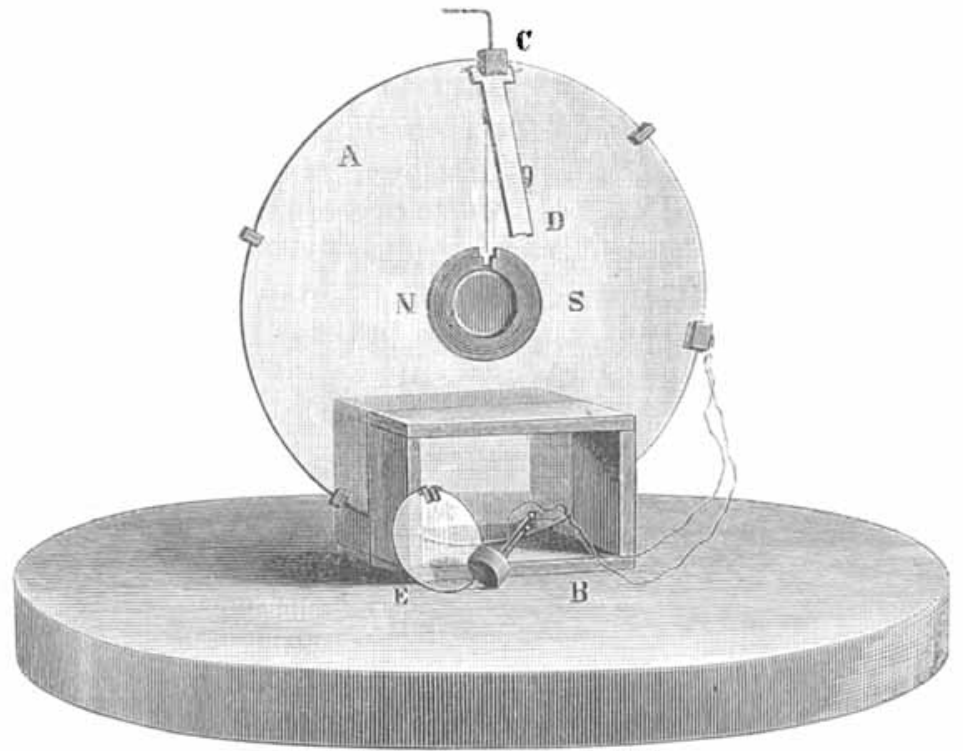

Fig. 1.

hält und bei gleicher Genauigkeit etwa den zehnten Theil der sonst gebräuchlichen Instrumente kostet. Dasselbe ist in Fig. 1 dargestellt.

Eine runde Glasplatte $A$ von $25 \mathrm{~cm}$ Durchmesser und $4 \mathrm{~mm}$ Dicke dreht sich in dem $4 \mathrm{~mm}$ breiten vertikalen Schlitz eines Holzrahmens $B$ von $12 \mathrm{~cm}$ Länge, $12 \mathrm{~cm}$ Breite und $8 \mathrm{~cm}$ Höhe. An ihrer höchsten Stelle trägt ein Reiter $C$ aus Hartgummi und Kork einen rechtwinkelig gebogenen Messing- 
drath mit einem Coconfaden und einem versilberten Hoblspiegel von $3,5 \mathrm{~cm}$ Durchmesser, auf dessen Rückseite ein Stahlring von $2 \mathrm{~cm}$ Durchmesser aufgekittet ist. Der Hohlspiegel schwebt in einer flachen, durch Plangläser geschlossenen, Kammer aus Hartgummi, in Mitten der runden Glasplatte, und wirft das Bild eines dünnen, von einer Petroleumflamme beleuchteten, Drahtes auf eine horizontale Millimeterscala. Die Lage des Bildes läst sich in einem hell erleuchteten Zimmer auf der 1 bis $2 \mathrm{~m}$ entfernten Scala bis auf $0,1 \mathrm{~mm}$ genau messen, wie bei der gewöhnlichen subjectiven Spiegelablesung.

Der Messingdraht mit Coconfaden und Spiegel wird gehoben oder gesenkt, der Holzrahmen durch einen untergeschobenen Holzkeil geneigt und die Glasplatte in dem Schlitz des Holzrahmens gedreht, bis der Coconfaden parallel seinem Spiegelbilde in der Glasplatte erscheint und der Spiegel frei schwebt. Durch Drehen des Messingdrathes im Reiter $C$ um einen vollen Kreis lässt sich in der üblichen Weise das Torsionsverhältniss des Coconfadens bestimmen, über den eine Hartgummirinne $C D$ geklappt wird, um ihn vor Luftströmungen zu schützen.

Das vordere Planglas $E$ der flachen Kammer hängt mit einer Nase aus Hartgummi in einem Ausschnitte des Hartgummiringes, kann leicht entfernt werden, und lehnt in der Zeichnung an dem Holzrahmen $B$. Die eingeklappte Fadenrinne sichert gleichzeitig die Lage des eingehängten Planglases $E$. Der Coconfaden läuft durch eine Rinne der Hartgummi-Nase.

Durch Luftdämpfung werden die Schwingungen des Hohlspiegels in 15 Sekunden beruhigt; oder in noch kürzerer Zeit, wenn man hinter dem Magneten an der Hinterwand der flachen Kammer einen Kupferpenny mit Wachs befestigt.

Die Tangentenbussole besteht aus einem seidebesponnenen Kupferdrahte von 0,27 mm Durchmesser, der in 1 oder 2 Windungen auf der Peripherie der runden Glasplatte $A$ mit 6 oder mehr Hartgummiklammern befestigt ist. Die Enden des Kupferdrahtes werden zusammengedreht, so dass sie in 20 oder $30 \mathrm{~cm}$ Abstand rom Drahtkreise mit angelötheten Stecknadeln in Quecksilbernäpfchen aus Kork eingesteckt werden können. 
Auf den Holzrahmen zu beiden Seiten der runden Glasplatte werden Drahtrollen aufgesetzt, wie bei einem gewöhnlichen Wiedemann'schen Multiplicator.

Der Draht der Tangentenbussole hat 0,5 Ohm Widerstand; 1 Sc. Ablenkung entspricht bei einer Drahtwindung und $1 \mathrm{~m}$ Spiegelabstand einer Stromstärke von 0,002 Amp. Eine Multiplicatorrolle von 135 Windungen hat 1 Ohm Widerstand und 1 Sc. entspricht 0,000005 Amp. (Rolle $F$ in Fig. 2.) Für starke Ströme stellt man einen Ring aus dickem

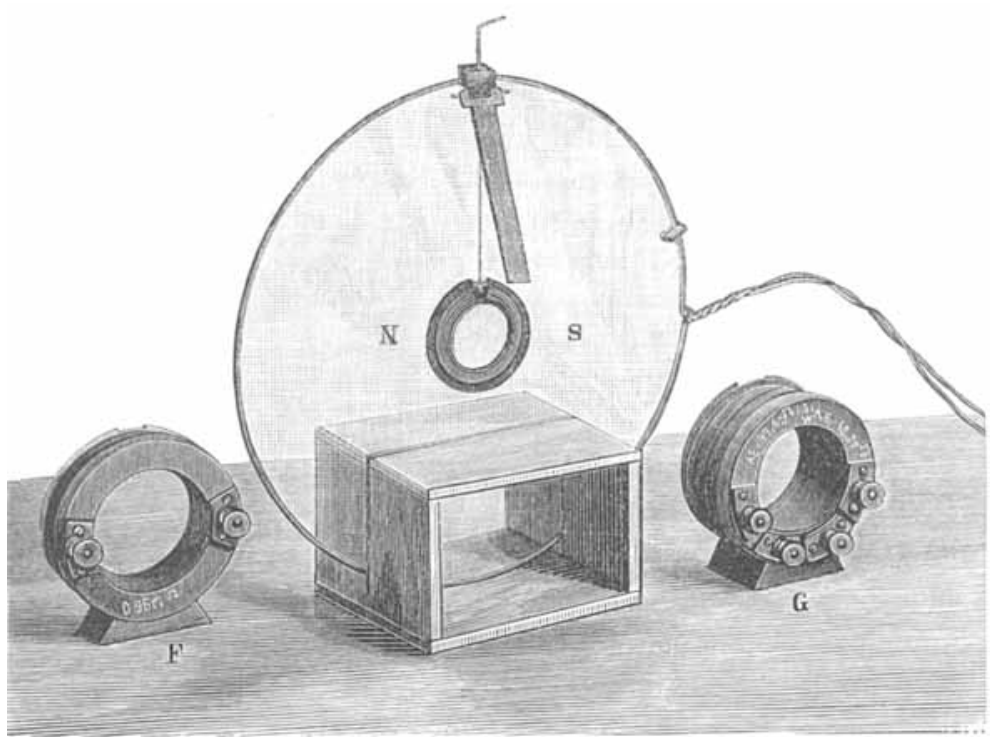

Fig. 2.

Kupferdrahte auf der Peripherie einer zweiten Glasplatte in passender Entfernung von dem Magnetometer auf, ähnlich wie bei einer Tangentenbussole von Helmholtz oder Gaugain.

Für einen Ring vom Radius $R$ in der Entfernung $E$ von der Magnetnadel ist die Stromstärke bei gleicher Ablenkung im Verhältniss $\left(1+E^{2} / R^{2}\right)^{3 / s}$ grösser, als mit der Magnetnadel in der Ringebene. Ein Drahtring von $25 \mathrm{~cm}$ Durchmesser muss in $17,34 \mathrm{~cm}$ oder $23,85 \mathrm{~cm}$ Entfernung gestellt werden, um ein Instrument von fünf- oder zehnmal geringerer Empfindlichkeit zu erhalten; Fig. 2 zeigt eine Tangentenbussole mit dickem Kupferdrahte, welche nach Entfernung der Magnet- 
nadel mit dem Apparate Fig. 1 combinirt eine Gaugain'sche Tangentenbussole von zehnmal kleinerer Empfindlichkeit gibt.

Als Hohlspiegel von 1 oder $2 \mathrm{~m}$ Radius werden biconvexe Brillengläser von 2 oder $4 \mathrm{~m}$ Brennweite (also von 0,5 oder 0,25 Dioptrien) verwandt, deren eine Seite versilbert und lackirt ist.

Für schwache Ströme stellt man neben die Magnetnadel Drahtrollen von 10000 Windungen oder wenn der Apparat als Differential-Multiplicator benutzt werden soll, Rollen von Doppeldraht mit 500 oder 1000 Windungen und 20 bis $50 \mathrm{Ohm}$ Widerstand. (Rolle $G$ Fig. 2).

Die Drahtenden der Tangentenbussole tragen angelöthete Stecknadeln von $4 \mathrm{~cm}$ Länge, welche in Quecksiłbernäpfchen aus Kork eingesetzt werden. Die Korkstücke sind mit Colophoniumkitt auf einer Glasplatte befestigt, und diese ist in einen niedrigen Pappkasten eingelegt, um das verschüttete Queck silber aufzunehmen. Eine kleine vertikale Glasplatte theilt ein solches Quecksilbernäptchen in 2 isolirte Hälften. Bei Umsetzen der Stecknadeln, die durch einen schwarzen und rothen Knopf zu unterscheiden sind, wird die Stromrichtung in der Tangentenbussole die entgegengesetzte.

An die freien Enden der mit den Multiplicatorrollen verknüpften dünnen Leitungsdrähte und an die übrigen Leitungsdrähte sind ebenfalls Stecknadeln angelöthet, um die Verbindungen schnell herstellen und lösen zu können.

Für schnelle Stromwechsel dienen Pohl'sche Stromwender mit 6 Korknäpfchen von $3 \mathrm{~cm}$ Abstand. Daneben sind noch andere Korknäpfchen in $3 \mathrm{~cm}$ Abstand festgekittet, um Widerstände von 1 bis $100 \mathrm{Ohm}$ aus Nickel-Mangan-Kupferdraht oder von 1000 bis $20000 \mathrm{Ohm}$ aus Graphit-bestrichenem Papier einlegen zu können. Das letztere wird in aufgeschnittene Bleikugeln von $8 \mathrm{~mm}$ Durchmesser geklemmt. Durch die Bleikugeln sind Stecknadeln gesteckt und festgelöthet. 2 angekittete Glasstreifen halten die Stecknadeln in $3 \mathrm{~cm}$ Abstand und schützen die Graphitschichten vor äusseren Einflüssen.

Zur Beleuchtung dienen Petroleumlampen mit Flachbrenner, über welche ein viereckiger Kasten aus dünnem geschwärzten Metallblech gestülpt ist. Der Kasten ist $26 \mathrm{~cm}$ hoch, $12 \mathrm{~cm}$ breit, $10 \mathrm{~cm}$ lang; oben und unten offen; trägt 
an der Hinterseite eine Oeffnung, durch welche der Docht regulirt wird; an der Vorderseite in Flammenhöhe einen Schlitz von $8 \mathrm{~mm}$ Breite und $40 \mathrm{~mm}$.Höhe, vor welchem ein verticaler Draht von $0,2 \mathrm{~mm}$ Durchmesser festgelöthet ist. Auf der Vorderseite des Kastens sind über dem Schlitze 2 kurze rechtwinkelig gebogene Messingdrähte aufgelöthet. In den von dieseu Messingdrähten gebildeten offenen Haken lässt sich ein horizontales Holzlineal mit der in $500 \mathrm{~mm}$ getheilten Papierscala leicht verschieben. Die Lampe wird so vor dem Hohlspiegel aufgestellt, dass ein scharfes Bild des dünnen vertikalen Metalldrahtes auf der Papierscala erscheint. Fig. 3 zeigt die

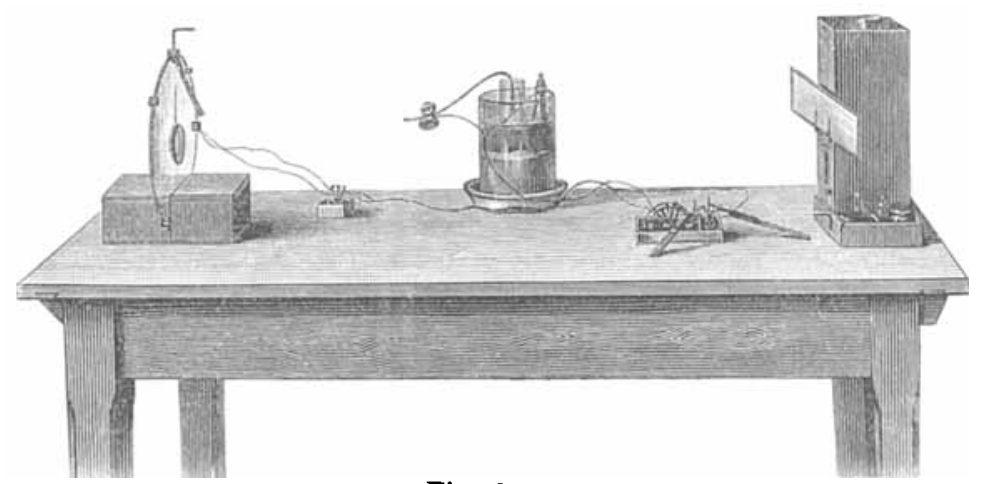

Fig. 3.

Aufstellung der Tangentenbussole mit Stromwendern, Kette und $2 \mathrm{Ohm}$ Widerstand im Stromkreis.

Die Apparate ${ }^{1}$ ) haben sich beim Gebrauch während mehrerer Jahre bewährt, sind leichter und schneller als die bisher gebräuchlichen aufzustellen, und lassen sich auch mit subjectiver Spiegelablesung benutzen.

b. Grosse Tangentenbussole mit Kreistheilung und Spiegelablesung.

In neuerer Zeit habe ich mit ähnlichen Mitteln aus Glas, Holz und Hartgummi grosse Tangentenbussolen construirt mit Oeldämpfung und einer Magnetnadel, deren Ablenkung nach

1) Zu beziehen durch Herrn Mechaniker Ad. Veith, Heidelberg, Hauptstrasse 15 . 
Belieben an einer Kreistheilung oder mit subjectiver oder objectiver Spiegelablesung gemessen werden kann.

Eine runde verticale Glasplatte $A$ (Fig. 4), von $40 \mathrm{~cm}$ Durchmesser, $4 \mathrm{~mm}$ Dicke und mit einem centralen Loch von $11 \mathrm{~cm}$ Durchmesser, dreht sich in dem $4 \mathrm{~mm}$ breiten und $55 \mathrm{~mm}$ tiefen Schlitz eines Holzrahmens $B$ von $20 \mathrm{~cm}$ Breite, $10 \mathrm{~cm}$ Höhe und $12 \mathrm{~cm}$ Länge. Ein rechtwinkelig gebogener

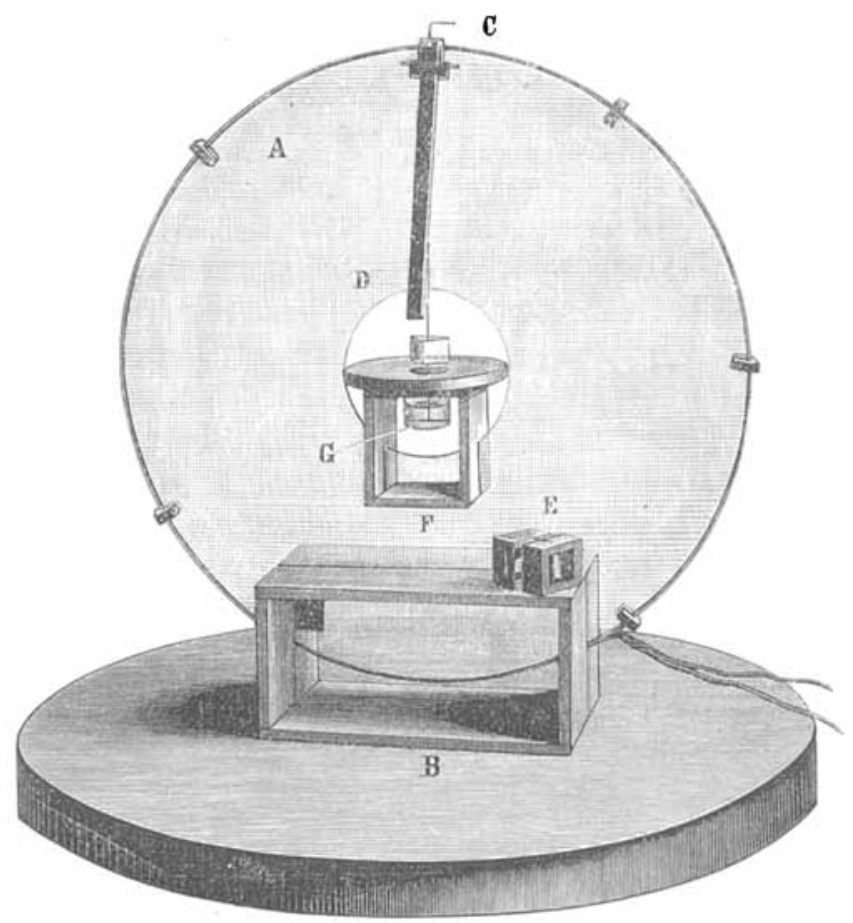

Fig. 4.

Messingdraht mit Coconfaden, Magnetnadel und Spiegel kann in einem Kork des Hartgummi-Reiters $C$ gehoben, gesenkt oder gedreht werden, wie bei dem oben beschriebenen Instrumente Fig. 1. Die gegen die Glasplatte $A$ geklappte Fadenrinne $C D$ aus Hartgummi und das zweitheilige Kästchen $E$ aus geschwärztem Carton mit 2 Glasfenstern von $20 \mathrm{~mm}$ Höhe und $26 \mathrm{~mm}$ Breite, schützen die Magnetnadel nebst Zubehör vor Luftströmmungen. Die Fenster stehen parallel der Glas- 
fläche $A$. Der untere Theil der Fadenrinne in der centralen Oeffnung bei $D$ ist $4 \mathrm{~mm}$ dicker, als der obere Theil, und wird durch ein Planglas geschlossen, das auf die Hinterfläche der Glasplatte $A$ aufgekittet ist.

Ein mit Seide besponnener Kupferdraht (von 0,27 oder $1,5 \mathrm{~mm}$ Durchmesser) wird mit 6 Hartgummiklammern auf der Peripherie der Glasplatte festgehalten und bildet den Drahtkreis der Tangentenbussole. In der centralen Oeffnung der Glasplatte $A$ ist ein kleiner Holzrahmen $F$ von $6,5 \mathrm{~cm}$ Breite und Länge und von $7,5 \mathrm{~cm}$ Höhe mit einem $4 \mathrm{~mm}$ breiten und $35 \mathrm{~mm}$ tiefen Schlitz aufgesetzt, so dass die obere quadratische Fläche des Holzrahmens ein horizontales Tischchen bildet,

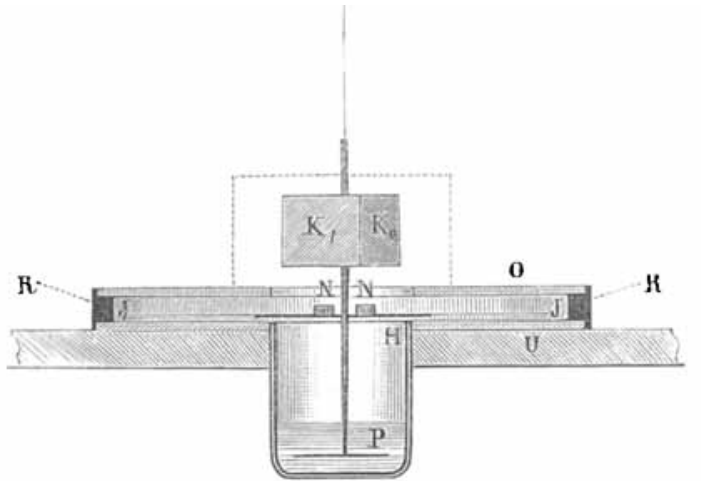

Fig. 5.

dessen Mitte in der Verlängerung des Coconfadens liegt. Die Holzrahmen $B$ und $F$ sind aus tannenen Brettchen von $7,5 \mathrm{~mm}$ Dicke mit Holznägeln zusammengefügt.

In einer centralen Oeffnung des Tischchens ist mit Hartgummi ein kleiner Glasbecher $G$ (Fig. 4 und 5) von $3 \mathrm{~cm}$ Durchmesser und $3,5 \mathrm{~cm}$ Höhe eingepasst, dessen gerader cylindrischer Rand $2 \mathrm{~mm}$ über die Tischfläche hervorragt und in das centrale Loch der runden Spiegelglasplatte $U$ eingreift, welche die Kreistheilung von $10 \mathrm{~cm}$ Durchmesser trägt. Auf dem Rande der Spiegelglasplatte $U$ ruht ein oben und unten ausgedrehter Messingring $R$ von $7,5 \mathrm{~mm}$ Höhe; auf diesem eine in zwei gleiche Hälften geschnittene Spiegelglasplatte $O$ mit centraler Oeffnung von $3 \mathrm{~cm}$. Diese Spiegelglasplatte bildet 
die obere, die Kreistheilung die untere Basis der flachen Büchse, in welcher die Magnetnadel $N$ schwebt, im Mittelpunkte des Drahtkreises und der Glasplatte $A$.

Der verticale weiche Kupferdraht am unteren Ende des Coconfadens trägt aufgelöthet ein horizontales Kreuz $H$ und eine runde Scheibe $P$ von $2 \mathrm{~cm}$ Durchmesser aus dünnem Kupferblech. Auf dem Kreuz sind 2 kleine Stahlmagnete $N N$ (von $12 \times 4 \times 2 \mathrm{~mm}$ ) und 2 Glasfäden $J$ senkrecht zur magnetischen Axe der Stahlmagnete aufgekittet. Das Kreuz ruht auf der Kreistheilung, wenn der Coconfaden gesenkt wird, so dass der Apparat ohne Geführdung des Coconfadens und der Glasfäden transportirt werden kann. Die geschwärzten Enden der Glasfäden schweben über der Kreistheilung und bestimmen die Ablenkung der Magnetnadel bis auf $0: 1^{0}$ genau. Die Kupferscheibe $P$ schwebt in Paraffinoel oder Olivenoel, mit welchem der Glasbecher $1 \mathrm{~cm}$ hoch gefüllt ist.

Die halbe Schwingungsdauer der Magnetnadel mit Spiegel und Gehänge beträgt 4 Sekunden. Die Nadel kommt nach 4 halben Schwingungen zur Ruhe. Durch Verkleinern der Kupferscheibe oder durch Zusatz von Petroleum zum Olivenoel kann man die Dämpfung verringern.

Als Spiegel dienen die 3 Seitenflächen eines rechtwinkeligen Prismas. 3 Silberspiegel, die mit Colophoniumkitt an den Kanten von 2 horizontalen Hartgummiplatten befestigt und mit diesen auf den oberen Theil des Kupferdrahtes aufgeschoben sind. Die Kathetenspiegel haben $20 \mathrm{~mm}$ Höhe und Breite und werden genau normal gestellt, indem man sie gegen die eine Kathetenfläche und ein Planglas auf der anderen Kathetenfläche eines rechtwinkeligen Glasprismas drückt, so lange der Kitt noch weich ist.

Der Hypotenusenspiegel von $28 \mathrm{~mm}$ Breite wird durch Drehung des ganzen Prismas parallel der magnetischen Axe der Magnetnadel gestellt, und zur Messung kleiner Ablenkungen dieser Nadel verwandt.

Die Kathetenspiegel $K_{1}$ und $K_{2}$ dienen dazu, Ablenkungen $\pm \varphi$ der Magnetnadel, welche nur wenig von $\pm 45^{\circ}$ verschieden sind, mit Spiegelablesung bis auf Minuten genau zu messen. Die Kathetenspiegel stehen während der Ablenkung dann nahezu parallel der Glasplatte $d$ oder dem magnetischen Meridian. 
Sie reflectiren denselben Scalentheil ins Fernrohr, wenn die Ablenkung genau $\pm 45^{\circ}$ beträgt. Reflectiren sie bei gleicher und entgegengesetzter Stromstärke die Scalentheile $s_{1}$ und $s_{2}$ in das Fernrohr, so würde die Ablenkung

$$
\pm \varphi=45 \pm \frac{s_{1}-s_{2}}{4 r} \frac{360}{2} \frac{\pi}{\pi}
$$

sein, wenn $r$ den Abstand der Scala vom Spiegel bezeichnet. Das obere oder untere Vorzeichen der rechten Seite gilt, wenn bei abnehmender Stromstärke $s_{1}$ abnimmt und $s_{2}$ zunimmt, oder umgekehrt.

Bilden die beiden Kathetenspiegel einen Winkel. der um $v$ grösser oder kleiner ist, als ein rechter, so würde $z u 45^{\circ}$ auf der rechten Seite der Gleichung (1) noch der Winkel $-v / 2$ oder $+v / 2$ zu addiren sein.

Den Winkel $90 \pm \nu$ der Kathetenspiegel kann man entweder mit dem Goniometer messen, oder mit folgendem Verfahren.

Man drückt ein auf der Hinterseite geschwärztes Planglas (Objectträger) gegen den einen Kathetenspiegel und betrachtet in dem aus dem Planglas und dem anderen Kathetenspiegel gebildeten Winkelspiegel die Bilder einer Lichtlinie (eines vertikalen Platindrahtes von $0,2 \mathrm{~mm}$ Durchmesser in einer Bunsen'schen Gasflamme). Sind die beiden durch Doppelreflexion im Winkelspiegel entstehenden Bilder der Lichtlinie um die Strecke $\sigma$ von einander und um die Strecke $\varrho$ vom Spiegel entfernt, so ist die Neigung der Kathetenspiegel

$$
\psi=90 \pm \nu=90 \pm \frac{\sigma}{4 \varrho} \frac{360}{2 \pi}
$$

das obere oder untere Vorzeichen gilt, je nachdem beim Verkleinern des Winkels $\psi$ (durch Einschiebung eines Keiles oder eines Kartenblattes zwischen Kathetenspiegel und Objectträger) die Bilder der Lichtlinien auseinandergehen oder zusammenrücken.

Sehr bequem ist es, bei diesem Versuche 2 Lichtlinien (zwei parallele Platindrähte in den beiden Seiten einer Bunsen'schen Gasflamme) vom Abstande $\sigma$ zu benutzen, und den Abstand $\varrho$ so zu wählen, dass das rechte Bild des einen Platindrahtes mit dem linken des anderen sich deckt. Die Entfernung der 
beiden Bilder einer Lichtlinie ist dann gleich dem Abstande der beiden Lichtlinien. Die Entfernung $\varrho$ der Lichtlinie vom Winkelspiegel wird mit einem Bandmasse gemessen.

Die Spiegel schweben in dem geschwärzten Cartonkästchen $E$, das auf der halbirten Spiegelplatte $O$ rubt und in Fig. 4 an einer andern Stelle auf dem Holzrahmen $B$ steht. Das eine Fenster des Kästchens ist durch ein Planglas, das andere durch ein biconvexes Brillenglas von 1 oder $2 \mathrm{~m}$ Brennweite geschlossen. - Für subjective Spiegelablesung wird das erstere, für objective Spiegelablesung das letztere benutzt, indem man die Petroleunlampe mit Gehäuse und Scala in 1 oder $2 \mathrm{~m}$ Entfernung von der Linse aufstellt.

Die Kathetenspiegel haben den Vortheil, dass man den Ablenkungswinkel $\varphi$ sehr genau messen kann für Stromstärken, bei welchen der Apparat die grösste Empfindlichkeit besitzt. Die Stromstärke beträgt dann bei den angegebenen Dimensionen wenig mehr als $6 \mathrm{Amp}$. Benutzt man nur 1 Spiegel so würde $1 \mathrm{Sc}$. bei $1 \mathrm{~m}$ Spiegelabstand einer Stromstärke von 0,0032 Amp. entsprechen.

Uebrigens habe ich auch Apparate mit Glasplatte $A$ von $30 \mathrm{~cm}$ Durchmesser, also einer $4 / 3 \mathrm{Mal}$ grösseren Empfindlichkeit, gebaut, bei denen Holzrahmen, Kreistheilung, Magnetnadel und Zubehör dieselben Dimensionen, wie bei dem grösseren Apparate, hatten.

Herrn H. Rönne, Assistenten am hiesigen physikalischen Institut, spreche ich für seine umsichtige Hülfe bei Herstellung dieser Tangentenbussole mit Kreistheilung und Winkelspiegel meinen besten Dank aus.

Heidelberg, den 27. October 1892. 